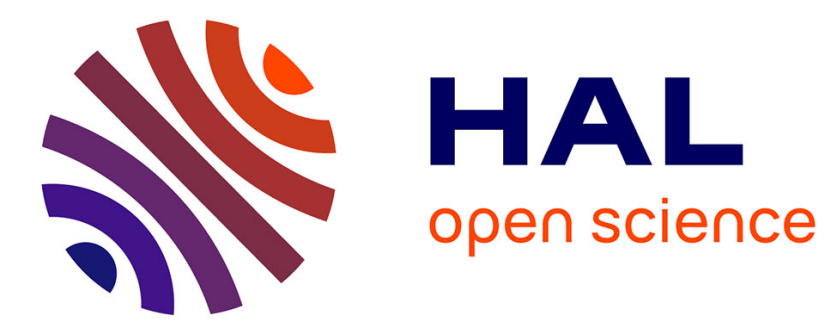

\title{
Probing Planck's law at home
}

Isabelle Bonnet, J. Gabelli

\section{To cite this version:}

Isabelle Bonnet, J. Gabelli. Probing Planck's law at home. European Journal of Physics, 2010, 31

(6), pp.1463-1471. 10.1088/0143-0807/31/6/012 . hal-02454261

\section{HAL Id: hal-02454261 \\ https://hal.science/hal-02454261}

Submitted on 30 Jan 2020

HAL is a multi-disciplinary open access archive for the deposit and dissemination of scientific research documents, whether they are published or not. The documents may come from teaching and research institutions in France or abroad, or from public or private research centers.
L'archive ouverte pluridisciplinaire HAL, est destinée au dépôt et à la diffusion de documents scientifiques de niveau recherche, publiés ou non, émanant des établissements d'enseignement et de recherche français ou étrangers, des laboratoires publics ou privés. 


\title{
Probing Planck's law at home.
}

\author{
I. Bonnet ${ }^{1} \ddagger$ and J. Gabelli. ${ }^{2}$
}

1 Laboratoire Matière et Systèmes Complexes, Université Paris Diderot, CNRS UMR 7057, 10 rue A. Domon et L. Duquet, 75013 Paris, France, EU

2 Laboratoire de Physique des Solides, Bât. 510, Université Paris Sud, CNRS - UMR 8502, 91405 Orsay, France, EU

E-mail: isabelle.bonnet@curie.fr, gabelli@lps.u-psud.fr

\begin{abstract}
We report on physics around an incandescent lamp. Using a consumer grade digital camera, we combine electrical and optical measurements to explore Planck's law of the black body radiation. This simple teaching experiment is successfully used to measure both Stefan's and Planck's constants. Our measurements lead to a strikingly accurate value for Planck's constant: $h=6.7 \pm 0.4 \times 10^{-34} \mathrm{~kg} \cdot \mathrm{m}^{2} \cdot \mathrm{s}^{-1}$. A digital camera is thus a sufficiently good equipment to measure a constant directly related to quantum mechanics.
\end{abstract}

\section{Introduction}

Spectroscopy, the study of the interaction between radiation and matter, has been used for almost two centuries to provide information about the matter under investigation. Each chemical element produces its own unique set of spectral lines serving as a fingerprint. In the middle of the nineteenth century, Kirchhoff was so able to prove that the Sun contains such familiar elements as sodium, magnesium, iron or copper by experimentally evidencing the positions of Fraunhofer's dark lines in a continuous spectrum. Behind these characteristic lines was hidden an universal law related to the continuous spectrum [1]. Following his research on the nature of spectral absorption, Kirchhoff was indeed led to introduce the concept of black-body, a body that is both a perfect emitter and absorber of heat radiation [2]. Even though it is slightly confusing for students allowing that it does not appear black, the name of black-body has been kept for historical reasons. One should rather talk about body in equilibrium with thermal radiations. It turns out that the radiated energy distribution of a black-body is a curve that depends only on the temperature $T$ and not on the nature and shape of the emitter.

The measurement of this spectral distribution and the difficulties to interpret it led Planck to come up 40 years later with the idea of energy quantization. He stated that exchange of energy between matter and radiation occurs in a discrete manner unlike

$\ddagger$ Present address: Institut Curie, UMR 3215 CNRS and Inserm U934, 26 rue d’Ulm, 75005 Paris, France, EU 
the classical assumption of continuous exchange. This marked the birth of quantum mechanics and its fundamental constant $h$, also called the Planck's constant. Expressed in SI units, the suitable units for everyday human experiments, $h \simeq 6.62 \times 10^{-34} \mathrm{~J}$.s. The smallest amount of energy exchanged by an oscillator at frequency $f$ is then set by $\delta E=h f . h$ is one of the smallest fundamental constants used in physics which explains that its existence had been ignored prior to Planck's work.

The main objective of this paper is to demonstrate how a digital camera and a simple flash light open the possibility of measuring such a small constant. Following Planck, we propose a simple experiment that can be achieved at home by measuring the light intensity emitted from a tungsten bulb. After a theoretical reminder on black-body radiation (section 2) we describe our setup (section 3). Section 4 then describes electrical and optical measurements that together lead to an estimate of Stefan's and Planck's constants at home.

\section{Black-body radiation theory}

\subsection{The black-body model}

Quantum revolution came from the inability to understand the spectrum of light emitted by hot bodies, what is called black-body radiation. An ideal black-body refers to an idealized object that absorbs all incident light at all wavelengths: no transmission and no reflection. The body is thus at thermal equilibrium with the electromagnetic field at the same temperature. Consequently, black-body radiation is the reference thermodynamic equilibrium state of light. In the final years of the nineteenth century, experimentalists measured, for different temperatures, the energy density of the radiation emitted by a black-body at various wavelengths [3]. The emitted spectrum only depends on the absolute temperature of the body and displays a broad peak that shifts from red to blue as the temperature increases (Figure 1).

Physicists failed for a long time to provide a theoretical explanation for this black-body radiation spectrum. The Rayleigh-Jeans law first tempted to describe the energy density $u(\lambda, T)$ of black-body radiation as a function of wavelength $\lambda$ for a fixed temperature $T$ eq. (1) $[4,5]$. It is based on a classical description of electromagnetic modes described by Maxwell theory. Indeed, the equipartition theorem of classical statistical mechanics states that all harmonic oscillator modes of a system at equilibrium have an average energy of $k_{B} T$, where $k_{B}$ is the Boltzmann constant $\left(k_{B}=1.38 \times 10^{-23} \mathrm{~m}^{2} \cdot \mathrm{kg} . \mathrm{s}^{-2} \cdot \mathrm{K}^{-1}\right)$. Thus, the distribution of energy radiated by a black-body is uniform and given by:

$$
u_{\mathrm{RJ}}(\lambda, T)=\frac{2 c k_{B} T}{\lambda^{4}} \quad \text { for } \quad \lambda \gg \frac{h c}{k_{B} T}
$$

where $c$ is the light speed $\left(c=3 \times 10^{8} \mathrm{~m} \cdot \mathrm{s}^{-1}\right)$. This law reproduces well the experimental data for large wavelengths. However, it predicts that the total emitted power (integrated over all wavelengths) should be infinite due to the divergence at small wavelengths. This is called the ultraviolet catastrophe. 


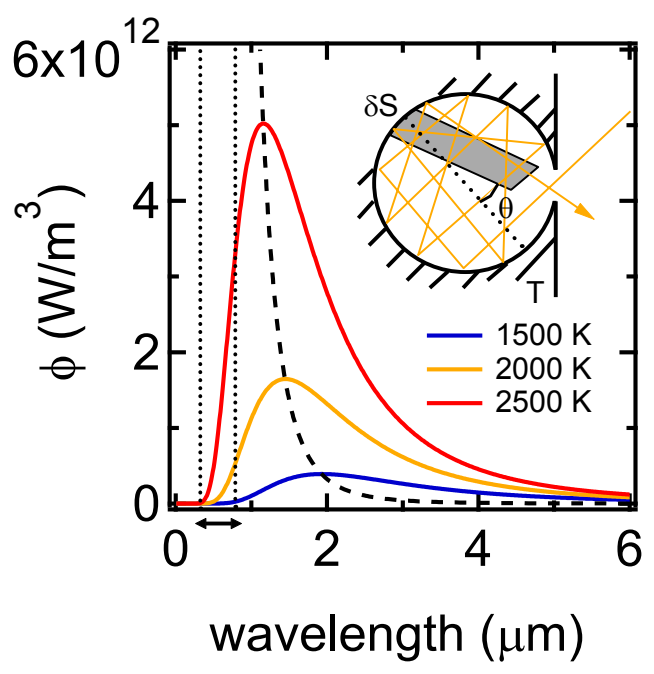

Figure 1. Black-body radiation spectrum. Radiation power $\phi$ as a function of wavelengths for three different temperatures: $T=1500 \mathrm{~K}$ (blue), $T=2000 \mathrm{~K}$ (orange) and $T=2500 \mathrm{~K}$ (red). Dashed curve corresponds to Wien's displacement law, $\lambda_{M}=2897 \mu \mathrm{m} / T[K]$. Vertical dotted lines denote the range of visible wavelengths. Inset: schematic of the model of black-body suggested by Wien and Lummer, a hollow sphere with internal reflecting walls and a narrow hole [3].

In 1893, Wien showed that the maximum radiation in the black-body spectrum displaces to the side of shorter wavelengths with increasing temperature which is called Wien's displacement law eq. (2, Figure 1)[6]:

$$
\lambda_{m} T=2897 \mu \mathrm{m} . \mathrm{K}
$$

where $\lambda_{m}$ is the wavelength of the maximum of the spectral density. Three years later, Wien proposed an empirical law [7] which succesfully describes experiments at short wavelengths and predicts of his displacement law:

$$
u_{\mathrm{W}}(\lambda, T)=\frac{k_{1}}{\lambda^{5}} e^{-\frac{k_{2}}{\lambda T}} \quad \text { for } \quad \lambda \ll \frac{h c}{k_{B} T}
$$

where $k_{1}$ and $k_{2}$ are constants determined experimentally. Even though Wien's law still failed at long wavelengths, its success at short ones indicated that already in 1896 that there was something amiss in classical physics. It was remaining to understand this discrepancy.

\subsection{Planck's law}

In 1901, Planck succeeded in obtaining an expression that perfectly agreed with experimental data at any wavelength [8]. He established the link between the classical expression of Rayleigh-Jeans and the phenomenological Wien expression. To obtain this result, he had to assume that the energy exchange between radiations (the electromagnetic field) and matter (the black-body) is done in finite amounts or quanta of energy. He introduced the Planck's constant $h=6.62 \times 10^{-34} \mathrm{~m}^{2} \cdot \mathrm{s}^{-1}$ giving the smallest 
amount of energy $\delta E$ carried by a mode of the electromagnetic field at wavelength $\lambda$ : $\delta E=h c / \lambda$. He ended up to describe the spectral energy density $u(\lambda, T)$ of a black body as a function of wavelength $\lambda$ at temperature $T$ :

$$
u_{\text {black body }}(\lambda, T)=\frac{8 \pi h c}{\lambda^{5}} \frac{1}{e^{\frac{h c}{\lambda k_{B} T}}-1}
$$

Given a black-body at temperature $T$, the quantity easily measurable on a bench is the radiated power $\phi$ (Figure 1) per unit surface area. In the case of a real body, its nature is only coded in its emissivity $\varepsilon$ which can be considered as a constant:

$$
u(\lambda, T)=\varepsilon u_{\text {black-body }}(\lambda, T)
$$

\subsection{Stefan's law}

As early as 1879 Stefan first determined the relation between the amount of power per surface unit $\Phi$ radiated by a body and its temperature. From experimental evidence, he stated that $\Phi$ varies as the fourth power of its absolute temperature [9]:

$$
\Phi(T)=\varepsilon \sigma T^{4}
$$

Here, $\sigma$ is Stefan's constant, equal to $5.67 \times 10^{-8} \mathrm{~W} \cdot \mathrm{K}^{-4} \cdot \mathrm{m}^{-2}$ and $\varepsilon$ the emissivity of the body which is 1 in case of a perfect black-body. This experimental fact can be deduced once the Planck's formula is known. Indeed, by considering energy radiated perpendicular to a small area $\delta S$, it must be noted that half of the energy density in the waves is going toward the walls $(u / 2)$. The radiated power being carried by photons at light speed $c$, the relation between power $\phi$ radiating per unit surface and wavelength through $\delta S$ during time $\delta t$ and energy $u$ is given by: $\phi \delta S \delta t=(c \delta S \delta t) u / 2$. Evaluating now the power seen at a given direction (angle $\theta$ in Figure 1), the effective area will be $\delta S \cos \theta$ and the effective speed will be $c \cos \theta$. The radiated power averaged over all angles will be reduced to :

$$
\phi(\lambda, T)=\int_{0}^{\pi / 2} d \theta c \cos ^{2} \theta \frac{u(\lambda, T)}{2}=\frac{c}{4} u(\lambda, T)
$$

To obtain the total power $\Phi$ radiated per unit surface area of a black-body at a temperature $T$, we integrate the Planck's law over wavelength and recover eq. (5).

\subsection{A filament as a black-body radiator}

In a light bulb, the Joule power dissipation, given by $P_{J}=U^{2} / R$ where $R$ is the resistance of the metallic filament, heats up the filament. Assuming that the filament behaves approximately as a black-body radiator (within a very wide band of electromagnetic radiation wavelengths), we can illustrate the connection between radiated power $P_{\text {rad }}$ and Joule dissipated power $P_{\mathrm{J}}$. The perceived color of a bulb lamp is yellowish white: according to Wien's displacement law, the temperature of the filament is of order $T \sim 3000 \mathrm{~K}$. In this daily experiment, the bulb remains at a much 
lower temperature and does not burn out. That means we can neglect the thermal conduction $P_{\text {cond }}$ by the socket of the bulb:

$$
P_{\mathrm{J}}=P_{\text {rad }}+P_{\text {cond }} \sim P_{\text {rad }}
$$

In our case, the temperature of the filament is set by a DC bias voltage $U$ across the lamp. The filament reaches a constant temperature solution of the equation $S \Phi(T)=P_{J}$, where $S=2 \pi r \ell$ is the surface of the filament, supposed to be a cylinder of length $\ell$ and radius $r$. Using eqs. (6) and (8), we end up with a steady state temperature:

$$
T=\left(\frac{r U^{2}}{2 \varepsilon \sigma \ell^{2} \rho(T)}\right)^{1 / 4}
$$

where $\varepsilon$ is the emissivity of the tungsten about 0.3 . Its resistivity $\rho$ is a non linear function of the temperature [10]:

$$
\rho[\mathrm{n} \Omega . \mathrm{m}]=48.0\left(1+4.8297 \times 10^{-3} T[\mathrm{~K}]+1.663 \times 10^{-6} T[\mathrm{~K}]^{2}\right)
$$

According to eq. (9), the temperature of the filament is thus entirely controlled by the voltage $U$.

\section{Experimental set-up}

\subsection{Description}

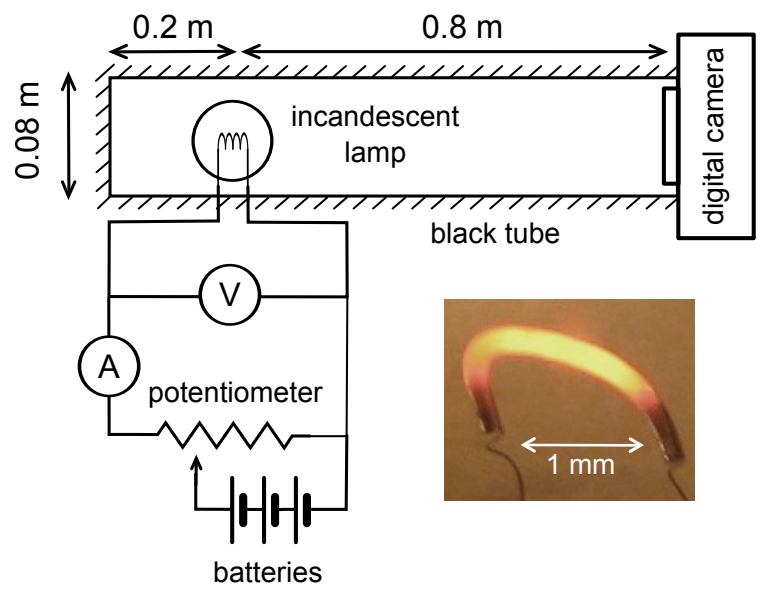

Figure 2. Schematic of the experimental set-up used to perform both electrical and optical measurements. A and V are amp- and voltage-meters respectively. Bottom right: picture of the flash light filament.

A blackened-inside cardboard tube of diameter $8 \mathrm{~cm}$ and length $1 \mathrm{~m}$ is used to realize darkness and avoid parasitic radiations from other sources. The camera and the bulb take place at the two tube extremities. The bulb comes from a $0.5 \mathrm{~W}$ flash light. The tungsten filament is biased by a voltage $U$ through a potentiometer and the association in series of three $4.5 \mathrm{~V}$ batteries. Voltage $U$ and current $I$ are measured with commercial Fluke 175 multimeters (Figure 2). Measurements require to take series of images with 


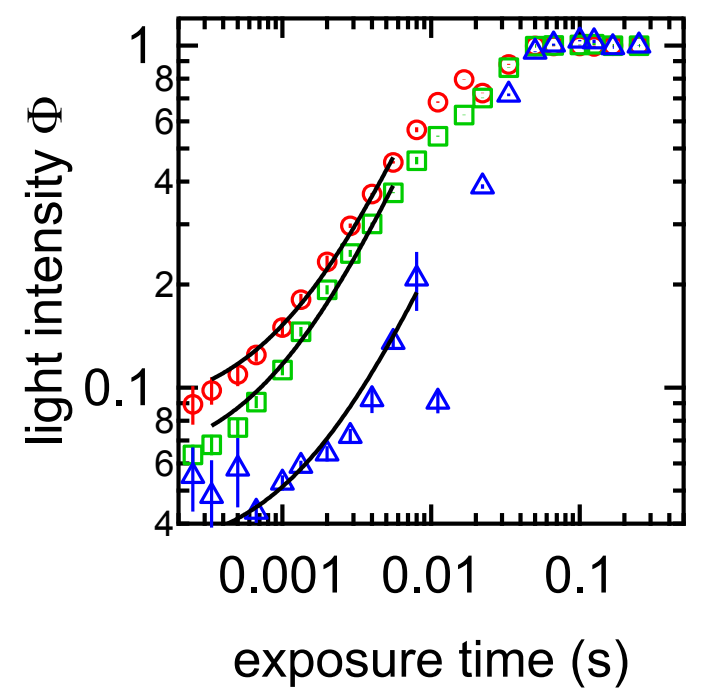

Figure 3. Intensity saturation of the camera detector as a function of the exposure time in a log scale for the three colour channels (RGB). The light source is the filament put at $0.5 \mathrm{~m}$ from the detector. The voltage is fixed at $U=10 \mathrm{~V}$. Black lines correspond to linear fits taking into account the background light.

different exposure times. To simplify them, we chose to control the camera remotely from a computer but it is possible to perform the measurements manually. The temperature $T$ of the filament is deduced from eq. (9).

\subsection{The digital camera}

The digital camera is a CCD (Coupled Charge Detector) image sensor that converts photons to electrons [11]. We used a Pentax K10D camera. Its detector consists of $10.75 \times 10^{6}$ photodetector elements called pixels of $6 \times 6 \mu \mathrm{m}^{2}$ size which record the level of light intensity from full black to white. Intensity of light denoted by $\phi$ is then the radiated power absorbed by a pixel during the exposure time. To detect color, colored filters are placed over each pixel assigning to it a primary color: the more common-used filters are the RGB (Red Green Blue). Every pixel will only have information about one color channel corresponding to the color of the filter in front of it. The camera produces images with 12 bit depth in a raw format [12]. The light intensity will be scaled taking $2^{12}=4096$ values between 0 and 1 .

\subsection{Calibration of the camera}

The camera was used without any lens and image acquisitions were conducted in a dark room. Acquisitions were done at room temperature $T_{0}=300 \mathrm{~K}$. In order to correctly interpret the light reaching each pixel, we did a calibration procedure. Parasitic reflections and angular dependance of absorption coefficient on the surface of the detector make light intensity $\phi(x, y)$ non homogeneous. To retrieve the flatness of the profile, we used one of the picture (exposure time $\tau=0.1 \mathrm{~s}$ and voltage $U=7.25 \mathrm{~V}$ ) as a reference 
$\phi_{0}(x, y)$. The factor $\phi_{0}(x, y) / \operatorname{Max}\left(\phi_{0}(x, y)\right)$, which is close to 1 , allows to define an intensity $\Phi(x, y)$ that does not depend on the position $(x, y)$ any more:

$$
\Phi(x, y)=\frac{\operatorname{Max}\left(\phi_{0}(x, y)\right)}{\phi_{0}(x, y)} \phi(x, y)
$$

The remaining fluctuations $\delta \Phi$ around the mean value over the whole image $\langle\Phi\rangle_{x, y}$ is then due to electric and photonic noises which can be assumed to be gaussian.

In the following, the light intensity for a given set of parameters (voltage $U$, exposure time $\tau$ ) will be denoted by $\Phi_{i}$ with $i=\mathrm{R}, \mathrm{G}, \mathrm{B}$ corresponding to one color. $\Phi_{i}$ is equal to the mean value of the gaussian fit of $\Phi(x, y)$. To avoid sensor saturation we set the camera to its lowest sensitivity (ISO 100), which allows to reduce noise, but requires a longer exposure time. We then fixed the voltage $U=10 \mathrm{~V}$ and took a series of pictures by increasing the exposure $\tau$ from the minimum the camera is able to do $(\tau=1 / 4000 \mathrm{~s})$ to an exposure time a long enough so that all pixels were saturated in all colors $(\tau=0.3 \mathrm{~s})$. Above a given exposure time, the intensity exhibits a saturation (Figure 3 ) which allows to define a maximum intensity that should not be exceeded in the following measurements: we set $\Phi \leq 0.5$.

\subsection{Fresnel diffraction for calibration of the wavelength camera filters.}

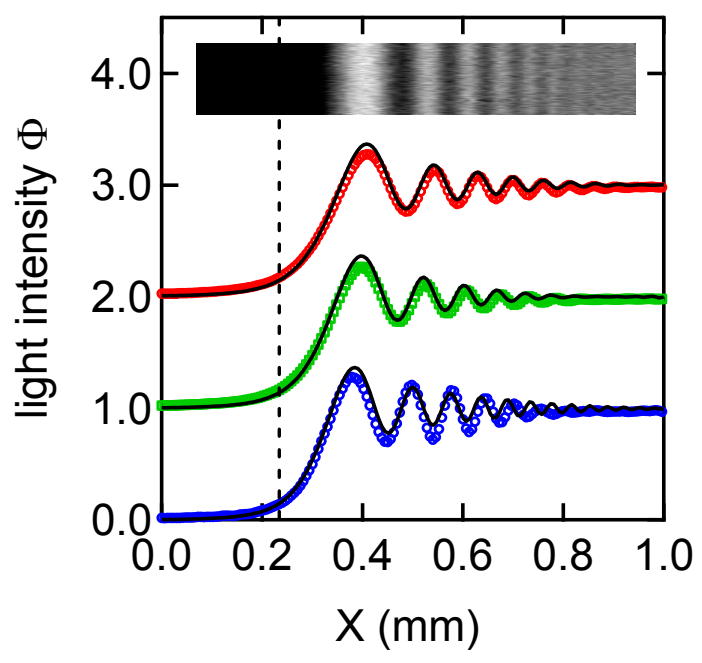

Figure 4. Diffraction by a straight edge (razor blade) in the Fresnel limit. Red, green and blue markers correspond to the three color layers. Curves have been vertically shifted for visibility. Black lines are fits by theoretical curves [13]. Dotted line indicates the edge of the razor blade. Inset: cropped picture of Fresnel's oscillations.

The determination of the central wavelengths $\lambda_{R, G, B}$ and widths $\delta \lambda_{R, G, B}$ of the RGB filters was performed with a Fresnel diffraction experiment on the edge of a razor blade [13] placed at distance $D=4.3 \mathrm{~cm}$ in front of the CCD sensor. It allows a direct comparison between the observed pattern of light and the prediction of diffraction theory 


\begin{tabular}{llll}
\hline Filters & Red & Green & Blue \\
\hline$\lambda(\mathrm{nm})$ & 650 & 557 & 464 \\
$\delta \lambda(\mathrm{nm})$ & 32 & 32 & 15 \\
\hline
\end{tabular}

Table 1. Center $\lambda$ and width $\delta \lambda$ wavelengths of the RGB filters fitted to the Fresnel diffraction experiment.

in the near-field region. It yields a pretty good estimate wavelengths of the different filters. We used the filament as a point light source (size $\sim 1 \mathrm{~mm}$ ) placed at a large distance $\sim 4 \mathrm{~m}$ from the diffracting edge. In that case, the diffracting pattern is given by the Fresnel integrals [14]: it only depends on the wavelength $\lambda$ of the radiations and the distance $D$ between the straight edge and the observation plane. We recorded the illumination pattern and verified the intensity formulas of the Fresnel diffraction of a straight edge (Figure 4). The agreement with the theoretical pattern is remarkably good, fitted values of $\lambda_{R, G, B}$ and $\delta \lambda_{R, G, B}$ are listed in Table 1.

\section{Planck's constant estimation}

\subsection{Stefan's law given by electrical measurement}

We first investigated the filament energy emission as a function of the temperature. Our aim was to test the $T^{4}$ dependence in Stefan law and to estimate the value of Stefan constant $\sigma$. In this experiment, the voltage $U$ across the lamp is increased by steps of $0.25 \mathrm{~V}$. At each voltage, the current $I$ through the lamp is recorded giving access to the resistance (inset of Figure 5) and to the Joule power $P_{J}=U I$. The temperature dependence of tungsten resistivity $\rho(T)$ is then used to extract the temperature of the filament eq. (9 [10]. Figure 5 displays the graph of $P_{\mathrm{J}}$ as a function of $T^{4}-T_{0}^{4}$. The linearity (the slope is equal to $\varepsilon S \sigma$ ) assesses the validity of Stefan's law. This is consistent with the hypothesis that the filament emits a black-body radiation without any thermal conduction loss. Estimating the surface of the filament $\left(S \sim 2 \pi r \ell \simeq 10^{-6} \mathrm{~m}^{2}\right.$, Figure 2 , bottom right), the slope $\simeq 1.560 \pm 0.005 \times 10^{-14}{\mathrm{~J} . \mathrm{K}^{-4}}^{-}$leads to:

$$
\sigma=5.52 \pm 0.01 \times 10^{-8} \mathrm{~W} \cdot \mathrm{K}^{-4} \cdot \mathrm{m}^{-2}
$$

This agrees with the tabulated value $\left(\sigma \simeq 5.67 \times 10^{-8} \mathrm{~W} \cdot \mathrm{K}^{-4} \cdot \mathrm{m}^{-2}\right)$ up to $3 \%$. This deviation can be attributed to the rough estimation of the surface of the filament.

\subsection{Wien's law given by optical measurement}

The semi log plot of the light intensity $\Phi_{i}$ as a function of $c / \lambda_{\mathrm{i}} k_{\mathrm{B}} T$ is shown in Figure 6 , where the wavelengths $\lambda_{i}$ are deduced from the Fresnel diffraction experiment described previously (see 3.4, Table 1). Measurements correspond to RGB intensities for voltage going from $0 \mathrm{~V}$ to $10 \mathrm{~V}$ : the set for $2 \mathrm{~V} \leq U \leq 10 \mathrm{~V}$ is performed with an exposure 


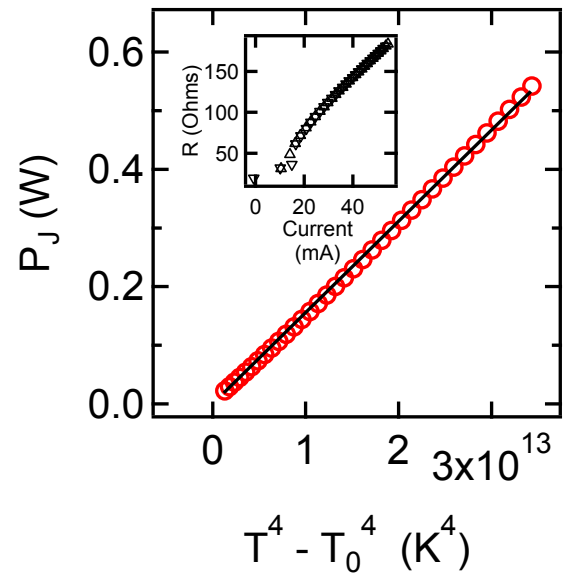

Figure 5. Stephan's Law: Joule dissipated power $P_{\mathrm{J}} v s . T^{4}-T_{0}^{4}$, where $T$ and $T_{0}$ are filament and room temperatures respectively. $T$ is tuned with the current through the lamp and deduced from the measurement of the filament resistance. Inset: resistance of the filament vs. current through it.

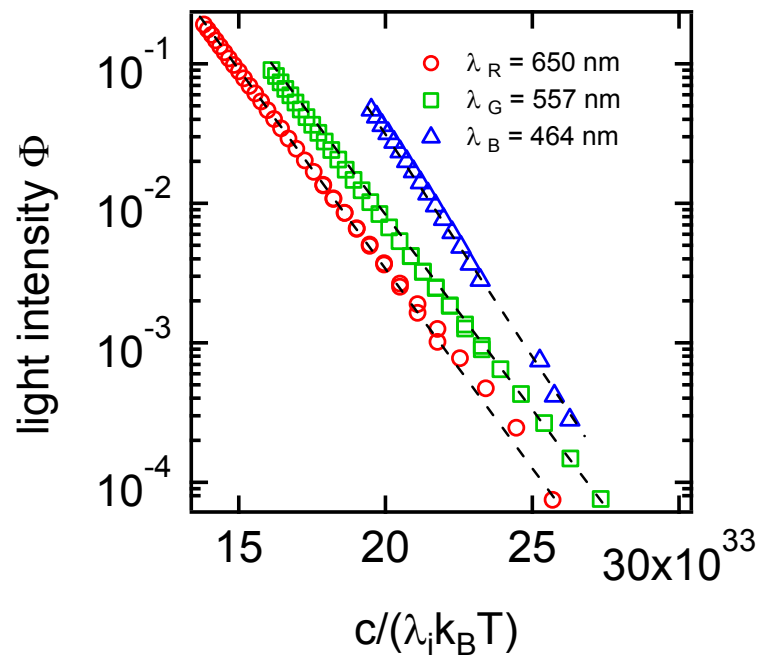

Figure 6. $h$ determination in the limit $h c /\left(\lambda_{i} k_{B} T\right) \gg 1$ : light intensity of the green (squares), blue (triangles) and red (circles) channels as a function of $c /\left(\lambda_{i} k_{B} T\right)$ in a semi $\log$ scale when the voltage is tuned from $3 \mathrm{~V}$ to $8 \mathrm{~V}$. Dotted lines indicate eq. 4.

time $\tau=1 / 10 \mathrm{~s}$, whereas another set, for $0 \mathrm{~V} \leq U \leq 5 \mathrm{~V}$, is performed with $\tau=1 \mathrm{~s}$ in order to use all the dynamics of the sensor. Data points fall on lines and according to eqs. $(3,4)$ their slopes correspond to the Planck's constant. Linear fittings for RGB color give respectively $6.52 \times 10^{-34}, 6.50 \times 10^{-34} \mathrm{~kg} \cdot \mathrm{m}^{2} . \mathrm{s}^{-1}$ and $7.23 \times 10^{-34}$ for the slopes. Averaging these values, we end up with:

$$
h=6.7 \pm 0.4 \times 10^{-34} \mathrm{~kg} \cdot \mathrm{m}^{2} \cdot \mathrm{s}^{-1}
$$

We thus test Wien's law and measure $h$ with $6 \%$ precision. This is surprisingly close $(1 \%)$ to the tabulated value. 


\section{Conclusions}

We have illustrated that a consumer grade light bulb follows a conceptual object as a black-body. The portable experiments which are described in this paper can be easily taken to schools and even home since they do not require any specialized equipment. Moreover, they allow to measure with a remarkably good accuracy the fundamental constant of quantum mechanics: the Planck constant. Although electric lamps are doomed to disappear in the context of the Kyoto agreement, we have shown that they easily offer students to enhance simple physic concepts.

\subsection{Acknowledgments}

We are grateful to F. Graner and B. Reulet for their careful reading of the manuscript and suggestions. Image analysis were performed with ImageJ (http://rsbweb.nih.gov/ij/) and data analysis were done with Scilab (http://www.scilab.org/) which are public domain softwares.

\section{References}

[1] Fraunhofer, J. von, 1814, Denkschriften der Königlichen Akademie der Wissenschaften zu München, 5, 193-226 (in German).

[2] G. Kirchhoff, 1860, Ueber das Verhältniss zwischen dem Emissionsvermögen und dem Absorptionsvermögen fr Wärme und Licht. Ann. d. Physik u. Chemie 109, 275-301 (in German).

[3] O. Lummer, E. Pringsheim, 1900, Über die Strahlung des schwarzen Körpers für lange Wellen. Verh. d. Deutsch. Phys. Ges., 2, 163-180 (in German).

[4] Lord Rayleigh (J.W. Strutt), 1900, Remarks upon the law of complete radiation, Phil. Mag., 49, 539-540.

[5] Jeans J. H., 1905, A comparison between two theories of radiation, Nature, 72, 293-294.

[6] Wien W., 1894, Temperatur und entropie der Strahlung, Ann. d. Physik, 52, 132-165 (in German).

[7] Wien W., 1896, Ueber die Energieverteilung im Emissionsspectrum eines schwarzen Körpers, Ann. d. Physik, 58, 662-669 (in German).

[8] Planck M., 1901, Ueber das Gesetz der Energieverteilung im Normalspectrum, Ann. d. Physik, 4, 553-563 (in German).

[9] Stefan J., 1879, Über die Beziehung zwischen der Wärmestrahlung und der Temperatur, Wiener Ber. II,79, 391-428 (in German).

[10] Handbook of Chemistry and Physics, 90 ${ }^{\text {th }}$ Edition 2009-2010, Editor: David R. Lide.

[11] W.S. Boyle and G.E. Smith, 1970, Bell Systems Technical Journal, 49, 587. W.S. Boyle and G.E. Smith were two of the three laureates of the Physics Nobel Prize in 2009 for the "invention of an imaging semiconductor circuit: the CCD sensor".

[12] Raw image files, also called digital negatives have all of the information needed to create an image. The file extension used here is .DNG and can be converted as a 16 bit. TIFF file by using ImageJ software. The image processing is then performed with Scilab software.

[13] Fresnel A., 1815, Premier mémoire sur la diffraction de la lumière, Académie des sciences, http://www.academie-sciences.fr/Membres/in_memoriam/Fresnel/Fresnel_pdf /Mem1818_p339.pdf (in French). 
[14] The diffraction pattern at a straight edge is given by:

$$
\phi_{\mathrm{F}}(x, \lambda)=\phi_{0}\left\{\left(\frac{1}{2}+C\left(\sqrt{\frac{2}{\lambda D}} x\right)\right)^{2}+\left(\frac{1}{2}+S\left(\sqrt{\frac{2}{\lambda D}} x\right)\right)^{2}\right\}
$$

By using gaussian filtering modelization, the measured intensity can be fitted by:

$$
\Phi\left(x, \lambda_{i}, \delta \lambda_{i}\right)=\int_{-\infty}^{+\infty} \phi_{\mathrm{F}}(x, \lambda) \exp \left(-\frac{\left(\lambda-\lambda_{i}\right)^{2}}{2 \delta \lambda_{i}^{2}}\right) d \lambda
$$

where $C$ and $S$ denote Fresnel integrals: $C(u)=\int_{0}^{\infty} \cos \left(\frac{\pi}{2} u^{2}\right) d u$ and $S(u)=\int_{0}^{\infty} \sin \left(\frac{\pi}{2} u^{2}\right) d u$ 